\title{
A Survey on Routing Protocols and Simulation Analysis of WRP, DSR and AODV in Wireless Sensor Networks
}

\author{
Shaiful Alam Chowdhury, Md. Ashraf Uddin and Shahid Al Noor
}

\begin{abstract}
The field of communications networks continues to emerge, thus leading to the rapid growth in the field of wireless sensor networks (WSNs). WSNs are noticeably different from traditional Ad hoc networks. The conspicuous difference between WSNs and traditional Ad hoc networks is that they (WSNs) are strictly constrained by limited supply of energy, capacity of storage, power of computation, and bandwidth. Because of these constraints and random deployment in some cases, it has become a challenge for the researchers to design an unblemished routing layer protocol for wireless sensor networks. In this paper we studied the motivation and design issues for routing protocols in WSNs with the limitations of existing routing protocols for WSNs. We also used GloMoSim-2.03 to find the shortcomings of traditional ad hoc routing protocols (DSR, WRF, AODV) when those are applied to WSN and to find out certain specific scenarios (considering low traffic networks and high traffic networks) of WSN where it is better to employ an existing Ad hoc routing protocol rather than developing a new one.
\end{abstract}

Index Terms-AODV, DSR, Routing protocols, WRP.

\section{INTRODUCTION}

The recent progress in Micro-electro-mechanical system (MEMS), low-power analog and digital electronics and low-power RF design naturally leads to the deployment of low-power, large-scale and low-cost wireless sensor networks [1]-[3]. Different categories of sensors such as thermal, seismic, visual, radar and infrared may be used to construct WSNs, which are capable of use in surveillance applications in a wide variety of environments [4]. Each sensor in a wireless sensor network is usually outfitted with a radio transceiver to work as a communication device, a physically diminutive microcontroller, and a battery which works as the energy source. Various shapes are available for the sensors, ranging from shoe-box sized sensors down to the size of grain of dust, though true microscopic dimensions are yet to be designed [5]. One or more centralized control points are typically defined in a sensor network, known as sinks, which are able to receive the efficacious information generated by the source nodes in response to some specific events [3].

The progression of sensor networks was primarily induced by military applications such as battlefield surveillance [6]. Remote sensors could be employed to obliterate some of the

Manuscript received July 1, 2012, revised July 31, 2012.

Shaiful Alam Chowdhury is with Stamford Universiy Bangladesh (e-mail: msacbd@gmail.com).

Md. Ashraf Uddin is with University of Chittagong (e-mail: mdashrafbd@gmail.com).

Shaahid Al Noor is with Stamford Universiy Bangladesh (e-mail: shaahid.nmsu@gmail.com). jumbles that may arise in battlefield .These sensors might be deployed to amass unerring information about current battlefield situations and rendering felicitous information to the soldiers, weapons and vehicles in the battlefield [7]. WSNs have also found applications in the automobile industry, broadly in two categories. Firstly, sensors inside the vehicle can be interconnected to build a network in order to congregate data about the states of the vehicle and driver and then supply it to the end user or to a self-regulating control system [8]. Secondly, the vehicles can act as nodes themselves, to form WSNs, so that one vehicle can be informed in advance about the presence of other vehicles and their respective locations in the road, the presence of strollers crossing the road, and traffic jam [8]. Environmental observation and forecasting systems (EOFS) is another application of WSNs [9]. Typically EOFS are large-scale, distributed embedded systems in which data initially moves from distant sensors to collection stations using wireless channels and then to unified processing through wired links. In healthcare systems lots of new scopes have been opened up by the ameliorations of WSNs. We can foretell a future where the areas like home facilitation, intelligent nursing homes, and clinical experiment can be aided by medical systems using wireless sensor networks [10].

In most of the applications of WSNs the indispensable properties of a sensor node like limitations in energy, memory, computing power and transmission power become crucial. Thus, routing protocols in WSNs must be conscious of the fact that, the memory of the sensors should not be overflowed and energy consumption by the sensors should be minimal.

\section{EXISTING ROUTING TECHNIQUES FOR WSNS}

The limitations in computing power, storage capacity, and energy consumption caused the widespread ad-hoc routing protocols unfeasible for wireless sensor networks. That is why in recent years many routing algorithms have been suggested and developed [3]. According to the network structure and routing criteria, the routing techniques in WSNs can be divided into Flat routing, Hierarchical routing, Adaptive based routing, Multi-path based routing, Negotiation based routing and Query based routing [12]. The working techniques and limitations of the first three categories are described in the following sections.

\section{A. Flat Routing}

Every node performs equal roles in Flat network routing. Sequential Assignment Routing (SAR), Directed Diffusion, and Minimum Cost Forwarding Algorithm (MCFA) are examples of Flat network routing. SAR is the first protocol in 
WSNs which considers Quality of Services (QoS) with energy efficiency [11]. For routing, SAR considers three factors: energy resources, QoS on the paths, and the priority levels of the packets [14]. The SAR protocol creates different paths to bypass route re-calculation overhead in case of route failure. Multiple paths are created by developing multiple trees where the root of each tree is a one-hop neighbor of the sink. In order to select the path, each node has to remember the energy resources as well as additive QoS parameters related to each path [15]. Though the working technique of SAR assures fault-tolerance and easy recovery, this protocol might experience huge overhead because of maintaining the related information at each of the sensors especially when the number of nodes is huge [11].

Directed Diffusion is a data centric (DC) routing for wireless sensor networks. The basic idea of the DC model is to synthesize the data collected from various sources in order to eliminate data redundancy and reduce number of transmissions so that energy consumption is minimized [11], [12]. Advantages of Directed Diffusion are: since it is data centric, all the communications are neighbor to neighbor and do not use node addressing mechanism. Moreover each of the sensor nodes is capable in caching which is an advantage regarding energy efficiency and delay. Another reason of being energy efficient is, Directed Diffusion is an on demand protocol where global network topology does not need to be maintained. However, this protocol is not feasible for all applications since its data delivery model is query-driven. Directed Diffusion method will not be efficient in applications where the sink required data from the sources continuously. For example, the protocol based on Directed Diffusion method will not be suitable for environment monitoring [11].

The MCFA assumes that the routing direction is always toward the scheduled base station. That is why; a sensor does not need to have a unique ID. They do not even need to maintain a routing table; instead they maintain the estimated least cost path from them to the base station [12]. This protocol is very useful especially when the resources are limited. However the MCFA can only be applied to the networks where there is only a single sink and multiple sources. Moreover this protocol might suffer from node breakdown and equal-cost paths [15].

\section{B. Hierarchical Routing}

In hierarchical or clustered based routing sensor nodes do not play same roles in a network [3], [12]. Sensor nodes are usually defined as cluster heads and members of the clusters. The cluster heads collect, process, and transfer data from its members or other cluster heads [3]. Examples of clustered-based protocols are Low-energy adaptive clustering hierarchy (LEACH), Power-Efficient Gathering in Sensor Information Systems (PEGASIS), Threshold-sensitive energy-efficient sensor network (TEEN), and Adaptive periodic threshold-sensitive energy-efficient sensor network (APTEEN) [12], [16], [17], [18], [19].

LEACH is one of the most well known clustered based routing protocols for WSNs [11]. This protocol has self-organizing capability and the cluster heads or local base stations are selected randomly in order to allocate the energy load fairly among the sensors [16]. From simulation result it is found that LEACH is more efficient from energy consumption perspective compared to other traditional routing techniques and thus prolongs the system lifetime [3] [16]. But LEACH is single-hop routing and every node has to transmit data directly to the cluster-head or local base station - thus, it is not suitable to employ the LEACH protocol for a huge sized network. Moreover dynamic clustering or random selection of heads causes a lot of overhead which may lessen the achievement in case of energy efficiency [11].

PEGASIS is another cluster-based protocol which is a near-optimal chain-based protocol and with ameliorations over LEACH protocol. Simulation results show that PEGASIS is more efficient than LEACH in case of energy consumption because instead of constructing multiple clusters, PEGASIS constructs chains from sensor nodes so that every sensor node transmits and receives from a close neighbor and only one of the sensor nodes is picked from that chain in order to transmit to the sink [11], [12], [17]. However, PEGASIS encounters significant delay for some distant nodes on the chain and the network might suffer when the single leader become an impediment [11].

TEEN, especially designed for time-sensitive applications, applies a data-centric technique with cluster-based networks. In TEEN nearer sensor nodes construct clusters and this development continues until the sink or base station is obtained [11], [18]. After the construction of clusters, the cluster heads broadcast hard threshold and soft threshold to the sensor nodes. Hard threshold is the predefined least value for an attribute to induce a sensor node to start its transmission. Likewise, soft threshold is the value that is used to decide whether a significant change occurred in the sensed attribute so that data transmission should take place and thus reduced the number of data transmissions [11], [18]. However, the sensor nodes will never communicate if the threshold value is not reached, which makes this protocol unfeasible for the applications where continuous observation is needed [12].

APTEEN is an improvement over TEEN which collects periodic data with response to time-critical events. The architecture of APTEEN is similar to TEEN. After constructing the clusters, the cluster heads share the attributes, hard and soft threshold values, and schedule of transmissions to all sensor nodes [19]. However the energy consumption in APTEEN is greater than TEEN. Additional drawbacks including the need for developing and implementing threshold function, constructing clusters for multiple levels, and the increased overhead are noticeable [11].

\section{Adaptive Based Routing}

Sensor protocols for information via negotiation (SPIN), is a well known adaptive based routing, which is also a data-centric protocol [11], [12]. SPIN distributes all information to every node in a network considering every node can act as a base station which makes it possible to search data at any node and find the data at once [12]. In SPIN data are named by high-level captions which are also known as meta-data [20]. These meta-data are shared among the sensor nodes before any data transmission. Upon receiving new data each of the sensors broadcast the 
meta-data to their neighbors. After receiving the meta-data the neighbors first check whether they need the actual data or not. If they need it (the nodes that do not have the data) they collect the data by sending a request message [20]. This mechanism solves data redundancy problem and thus energy efficiency problem for wireless sensor networks. Additionally, if SPIN is used as a routing protocol in a network, the topological changes are localized as each of the sensor nodes only needs to know its single-hop neighbors [11]. However, one of the limitations of SPIN is that the meta-data technique cannot assure the data delivery in every case. If a node (destination) which is interested to retrieve data from a distant node (source) but other nodes between the source and destination nodes do not have interest in that specific data, such data will not be delivered to the destination node [11].

\section{EMPLOYING AD HOC ROUTING PROTOCOLS}

As we discussed in the previous section, none of the protocols is perfect for wireless sensor networks regarding energy efficiency and QoS. Furthermore, all of the protocols might suffer from security problems as security issues are not considered while designing them [21]. In this section three ad hoc routing protocols are discussed with their pros and cons. These are Wireless Routing Protocol (WRP), Dynamic Source Routing (DSR) and Ad hoc on Demand Distance-Vector (AODV). Then the results of employing these protocols in WSNs are shown to identify at least one of these protocols can be selected for some WSNs applications.

WRP is a proactive or table-driven protocol as it preserves the most recent view of the network and each of the sensor nodes has a route to all the destination nodes in advance [22]. The WRP maintains a set of four topology tables which are: distance table (DT), routing table (RT), link cost table (LCT), and message retransmission list (MRL) [22]. The network views of the neighbors are contained in DT, the recent view of network for known destination nodes are contained by RT, the cost for each link to transmit messages are maintained by LCT and the update messages that are to be retransmitted are contained by MRL. One of the noticeable advantages of WRP is, as the routes to all the destinations are ready in advance, the route setup time is reduced greatly. But the process of maintaining a list of topology tables requires more memory and complex processing ability. Because of excessive overhead involved in updating the tables, WRP is not feasible for highly mobile and large networks [22].

DSR is a reactive or on-demand protocol - it discovers a route only when it is asked by a node to communicate with a destination. The noticeable feature in DSR is it is beacon-less and that is why it does not need periodic HELLO packet transmissions which are used in other on-demand protocols by a node to inform the neighbors about its existence [22]. In DSR when a route to a destination is needed, the RouteRequest packets are flooded in the network. When the destination node gets the RouteRequest packet it responds by sending RouteReply packet back to the source. As this protocol does not need periodic update, it is not a bad choice to select DSR protocol for a network which is strictly constrained by energy consumption. However, the connection setup delay in DSR is higher than the proactive protocols and the performance of DSR is poor for a highly mobile network [22].

AODV is also an on-demand routing protocol like DSR. But the difference is it is not beacon-less. Moreover unlike DSR, the AODV uses destination sequence number, source sequence number, source identifier, destination identifier, broadcast identifier, and the time to live (TTL) field in order to select an up-to-date route to the destination node [22]. However, AODV is not suitable for energy and bandwidth sensitive applications as it needs periodic beaconing. Moreover the intermediate nodes might construct inconsistent paths if the source and destination sequence number are not up-to-date [22].

For our simulation we used GloMoSim-2.03. We used percentage of packet loss and energy consumption by the nodes as our performance metrics. The reason behind the selection of GloMoSim is it is a widely accepted and scalable environment especially for large networks. The analysis of the results found from the simulation is given below:

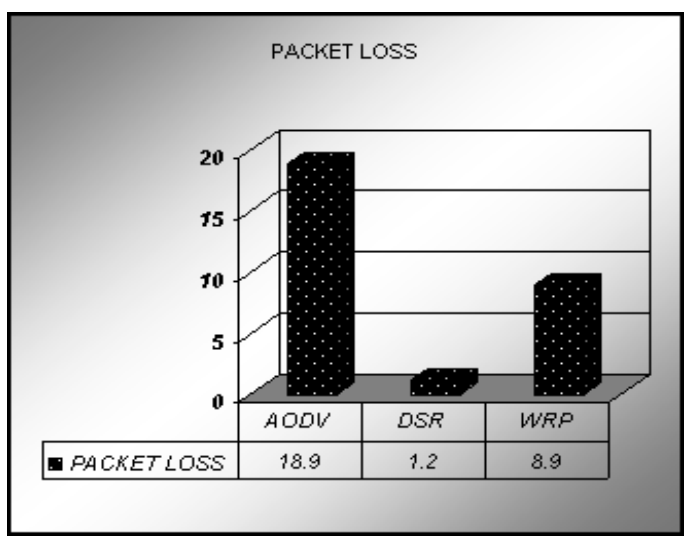

Fig. 1.(a): Percentage of Packet loss (50 nodes, 10 sources)

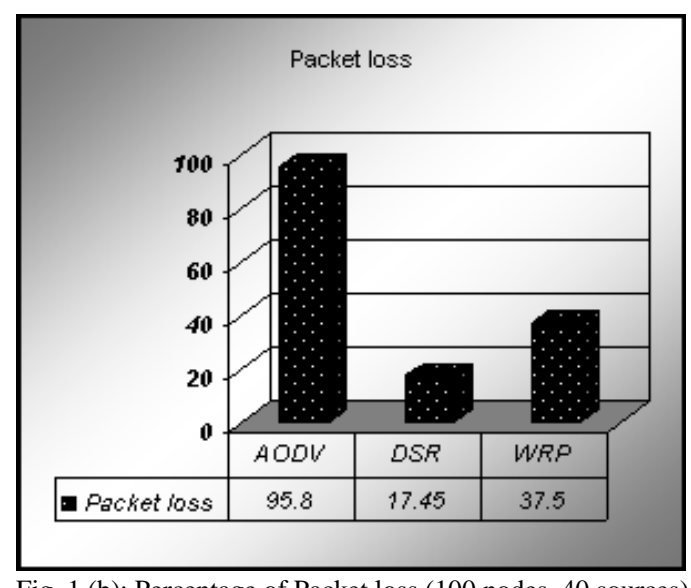

Fig. 1.(b): Percentage of Packet loss (100 nodes, 40 sources)

From Fig.1(a) and Fig.1(b) it can be seen that, the percentage of packet loss for DSR is very low both for low traffic and high traffic networks. WRP performs moderately in both cases. For generating periodically extra control packets (which might leads to memory overflow) and constructing inconsistent paths in some cases, the AODV protocol is outperformed by WRP and DSR. As we find from the above figures, DSR is the best protocol in case of packet loss while compared with WRP and AODV.

From Figure-2(a) and Figure 2(b) we find that DSR 
outperformed both the WRP and AODV routing protocol in case of energy consumption too. As it was expected, in case of energy consumption, for low traffic network WRP performs worst because of maintaining set of tables to setup routes in advance without necessity. But as it can be seen from Figure-2(a), surprisingly WRP outperforms AODV in case of energy consumption for high traffic network. One thing that might be responsible for that is the inconsistent paths (when AODV is used) constructed by the intermediate nodes in the network.

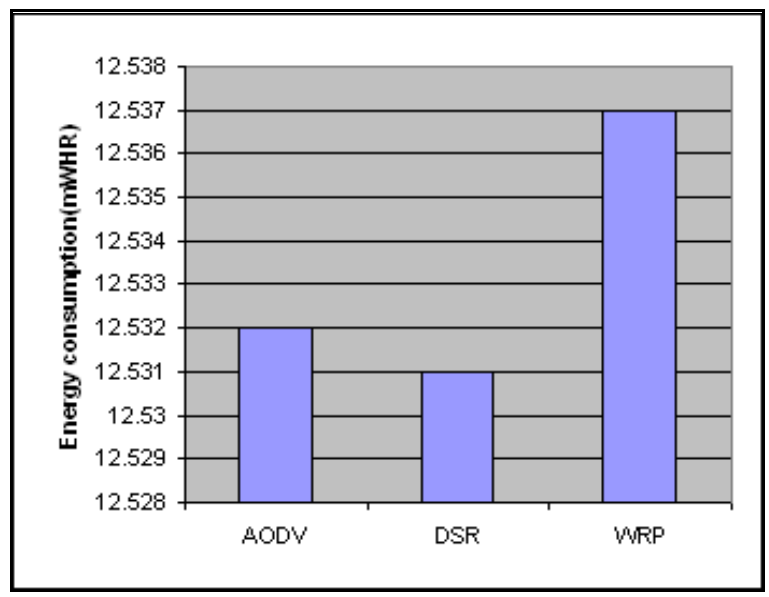

Fig. 2.(a): Energy consumption by the nodes (50 nodes, 10 sources)

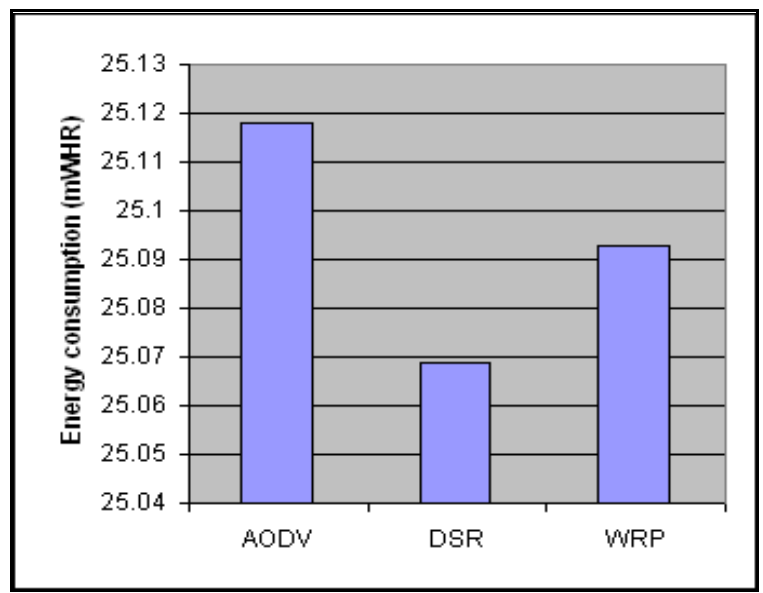

Fig. 2.(b): Energy consumption by the nodes (100 nodes, 40 sources)

\section{CONCLUSION AND FUTURE WORKS}

Since it is a very arduous and challenging task to design a perfect routing layer protocol for WSNs, in this paper we examined the results of employing traditional ad hoc routing protocols in WSNs. As it can be seen from our simulation results, the DSR protocol is not a bad choice for the WSNs applications where reliability and energy efficiency are crucial. But as, it is not a consummate solution to employ DSR in WSNs, it would be definitely a worthwhile research to design a flawless routing protocol for WSNs.

The problem is that the protocol has to solve some sophisticated problems like data redundancy, energy efficiency, and collisions between data without being sophisticated itself, because of the limitations in memory, computing power and energy resources. So applying an approximation algorithm for routing may be an optimal solution for the WSNs.

\section{ACKNOWLEDGEMENTS}

All the praise for Almighty God. This work has become true for His wishing and helping. I wish to express profound gratitude and sincere appreciation to Mr. Md. Rokan Uddin Faruqui, Assistant Professor of the Department of Computer Science and Engineering, Chittagong University, Chittagong, for his support and direction for this work.

\section{REFERENCES}

[1] D. Estrin "Embedded networked sensing research: Emerging systems challenges," In NSF Workshop on Distributed Communications and Signal Processing, Northwestern University, December 2002.

[2] D. Estrin, R. Govindan, J. Heidemann, and S. Kumar "Next century challenges: scalable coordination in sensor networks," ACM MobiCom'99, Washingtion, USA, 1999, pp. 263-270.

[3] C. Yin and A. Orooji "Routing Protocols for Sensor Networks," In Proceedings of ICWN' 2006. pp. 15-21.

[4] I. F. Akyildiz, W. Su, Y. Sankarasubramaniam, and E. Cayirci, "Wireless sensor networks: a survey," Computer Networks (Elsevier) Journal, vol. 38, no. 4, pp. 393-422, March 2002.

[5] S. A. Chowdhury, M. T. Islam, F. T. Jaigirdar, M. R. U. Faruqui, and S. A. Noor, "Performance study and simulation analysis of CSMA and IEEE 802.11 in Wireless Sensor Networks and limitations of IEEE 802.11," Proceedings of 12th International Conference on Computer and Information Technology, 21-23 December, 2009, Dhaka , Bangladesh , pp. 431-436.

[6] T. Arampatzis, J. Lygeros and S. Manesis, "A Survey of Applications of Wireless Sensors and Wireless Sensor Networks,” Mediterranean Conference on Control and Automation Limassol, pp. 719-724, June 27-29, 2005.

[7] A. Perrig, R. Szewczyk, V. Wen, D. culler, and J. Tygar, "SPINS: Security Protocols for Sensor Networks," In Seventh Annual ACM International Conference on Mobile Computing and Networks (Mobicom 2001), Rome Italy, July 2001.

[8] J. M. Ferro, L. M. Borges, F. J. Velez, Member, IAENG, and A. S. Lebres "Applications of Wireless Sensor Networks," Proceedings of the World Congress on Engineering 2007, vol. 2, July 2 - 4, 2007, London, U.K.

[9] D. Steere, A. Baptista, D. McNamee, C. Pu, and J. Walpole "Research challenges in environmental observation and forecasting systems," In ACM/IEEE MOBICOM '00, Boston, August 2000.

[10] J. A. Stankovic, Q. Cao, T. Doan, L. Fang, Z. He, R. Kiran, S. Lin, S. Son, R. Stoleru, and A. Wood "Wireless Sensor Networks for In-Home Healthcare: Potential and Challenges,” In HighConfidence Medical Device Software and Systems (HCMDSS) Workshop, Philadelphia, PA, June 2-3, 2005.

[11] K. Akkaya and M. Younis "A Survey of Routing Protocols in Wireless Sensor Networks," In the Elsevier Ad Hoc Network Journal, Vol. 3/3 pp. 325-349, 2005.

[12] J. N. Al-Karaki, A. E. Kamal. A Taxonomy of Routing Techniques in Wireless Sensor Networks. In Handbook of Sensor Networks: Compact Wireless and Wired Sensing Systems. CRC Press, Boca Raton, 2004.

[13] K. Romer and F. Mattern "The design space of wireless sensor networks,” IEEE Wireless Communications, 11(6):54-61, December 2004.

[14] K. Sohrabi and J. Pottie "Protocols for self-organization of a wireless sensor network,” IEEE Personal Communications, Volume 7, Issue 5, pp 16-27, 2000.

[15] W. D. Henderson and S.Tron "Verification of The Minimum Cost Forwarding Protocol for Wireless Sensor Networks," IEEE Conference on Emerging Technologies and Factory Automation, 20-22 Sept. 2006, pp. $194-201$.

[16] W. R. Heinzelman, A. Chandrakasan, and H. Balakrishnan "Energy-efficient communication protocols for wireless microsensor networks," In Proceedings of the Hawaii International Conference on Systems Sciences, Jan. 2000.

[17] S. Lindsey and C. Raghavendra "PEGASIS: power-efficient gathering in sensor information systems,” In IEEE Aerospace Conference, 2002.

[18] A. Manjeshwar and D. P. Agarwal "TEEN: a routing protocol for enhanced efficiency in wireless sensor networks,” In 1st Int. Workshop Parallel Distributed Computing Issues Wireless Networks Mobile Computing, April 2001. 
[19] A. Manjeshwar and D.P. Agrawal "APTEEN: a hybrid protocol for efficient routing and comprehensive information retrieval in wireless sensor networks," In Proceedings of the International Parallel and Distributed Processing Symposium, (IPDPS 2002), Ft. Lauderdale,FL, 15-19 April 2002. pp. 195-202.

[20] W. Heinzelman, J. Kulik, and H. Balakrishnan "Adaptive protocols for information dissemination in wireless sensor networks," In 5th Annual ACM/IEEE International Conference on Mobile Computing and Networking (MobiCom_99), Seattle, WA, August 1999.

[21] C. Karlof and D. Wagner "Secure routing in wireless sensor networks: Attacks and countermeasures," In First IEEE Int'lWorkshop on Sensor Network Protocols and Applications, May 2003.

[22] C. S. R. Murthy and B. S. Manoj "Routing Protocols for Ad Hoc Wireless Networks. In Ad Hoc Wireless Networks: Architectures and Protocols,” Dorling Kindersley (India), ISBN 81-317-0688-5.

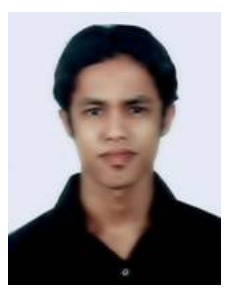

Shaiful Alam Chowdhury was born in Chittagong, Bangladesh on November 10, 1984. He is now a full time faculty member of Stamford University Bangladesh. He received his B.Sc. in Computer Science and Engineering (April, 2009) from University of Chittagong, Bangladesh securing $1^{\text {st }}$ position in the department. His current research interests are wireless sensor networks and algorithms.

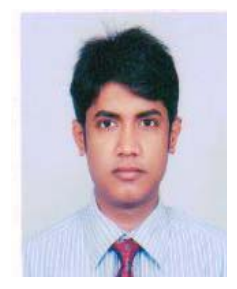

Md. Ashraf Uddin was born in Chittagong Bangladesh. He is currently an M.Sc student and received his B.Sc. in Computer Science and Engineering (April, 2009) from University of Chittagong Bangladesh. His current research interests are wireless sensor networks and AI.

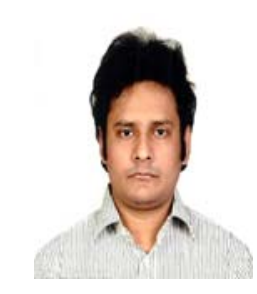

Shahid Al Noor is a full time faculty of Stamford University Bangladesh in the department of Computer Science. He was also a former faculty member of Prime University, Bangladesh in the department of Electronics and Telecommunication Engineering. He Obtained both of his MSc and BSc in Information and Communication Engineering from University of Rajshahi, Bangladesh in 2006 and 2005 respectively. His research interest includes Distributed Networks, Wireless Sensor Networks and Computer Vision and Natural Language Processing. 